\title{
Analyzing Top-Notch Innovative Competency of Postgraduate Students Based on Competency Model
}

\author{
Bing Bai, Zhiqiong Guo
}

Business School, Jiangsu Normal University, Xuzhou Jiangsu, 221116, China (joan_monday@163.com)

\begin{abstract}
This paper, taking the competency model as the theoretical basis, analyzes Top-Notch innovative competency of postgraduate students. On the basis of literature review of postgraduate competency, combining with expert interviews, we summarized four aspects that are knowledge, skills, character and personality which Top-Notch innovative postgraduate students should have, and in term of "iceberg" competency model we model "iceberg" competency model of Top-Notch innovative postgraduate students, which can be the foundation for follow-up study of capability evaluation.
\end{abstract}

Keywords — competency model, postgraduate students, top-notch innovative

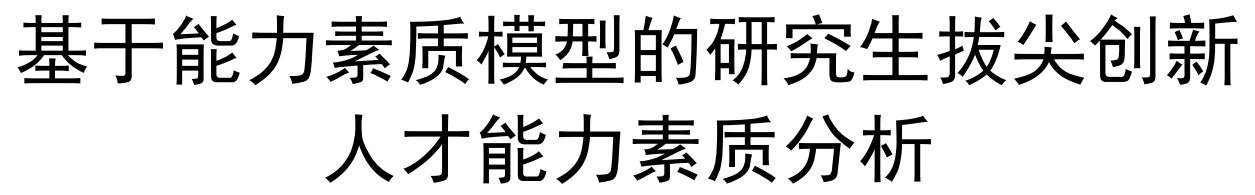

白冰 郭志琼

江苏师范大学商学院, 徐州, 江苏, 中国

摘 要 本文以能力素质模型为理论基础, 对研究生拔尖创新人才所应具备的能力素质进行了分析。在对研究生能力素质文献 分析基础之上, 结合专家访谈, 归纳总结出研究生拔尖创新人才能力特征应具备知识、技能、品质与个性等四个方面, 并依据能力 素质 “冰山” 模型, 建立了研究生拔尖创新人才能力素质冰山模型, 为后续的研究生拔尖创新人才能力测评奠定基础。

关键词 能力素质模型, 研究生, 拔尖创新

\section{1. 引言}

随着全球化进程不断加快, 知识经济日益显现, 现代 科技突飞猛进, 世界各国经济竞争和综合国力竞争愈加激 烈。我国要在 21 世纪的国际社会占有一定的地位和具有 较强的竞争实力, 就必须要有大批具有创新意识和创新能 力的人才。习总书记多次指出: 人才是发展经济的根本, 应该把人才的开发视为战略重点来抓。

研究生教育处于高等教育最高层次的, 担负着培养高 素质人才和发展科学技术的双重任务, 是培养高层次创新 人才的主渠道。高等院校如何加强研究生教育质量, 培养 更多具有创新能力和综合素质的拔尖人才, 更好地满足经 济建设、科技进步和社会发展需要是目前高校面临的一个 重要问题。本文以能力素质模型为基础, 对研究生拔尖创

国家社会科学基金教育学项目（No. CIA130172）
新人才能力构成进行系统剖析。

\section{2. 能力素质模型}

能力素质模型是将能力素质 (职业素养、能力和知识) 按内容、角色或是岗位有机地组合在一起, 职业素养、能 力和知识中的每项内容都会有相关的行为描述, 通过这可 观察、可衡量的行为描述来体现员工对于该项职业素养、 能力和知识的掌握程度。典型的能力素质模型是冰山模型 (Iceberg Competency Model)和洋苟模型。素质冰山模型由 麦克利兰提出, 他把人的素质模型形象地描绘成一座冰山, 冰山下的部分是我们所指的潜在的特征, 从上到下的深度 不同则表示被挖掘与感知的难易程度不同, 向下越深越不 容易被挖掘与感知。冰山上的部分是表象部分, 即人的知 识与技能, 容易被感知。 


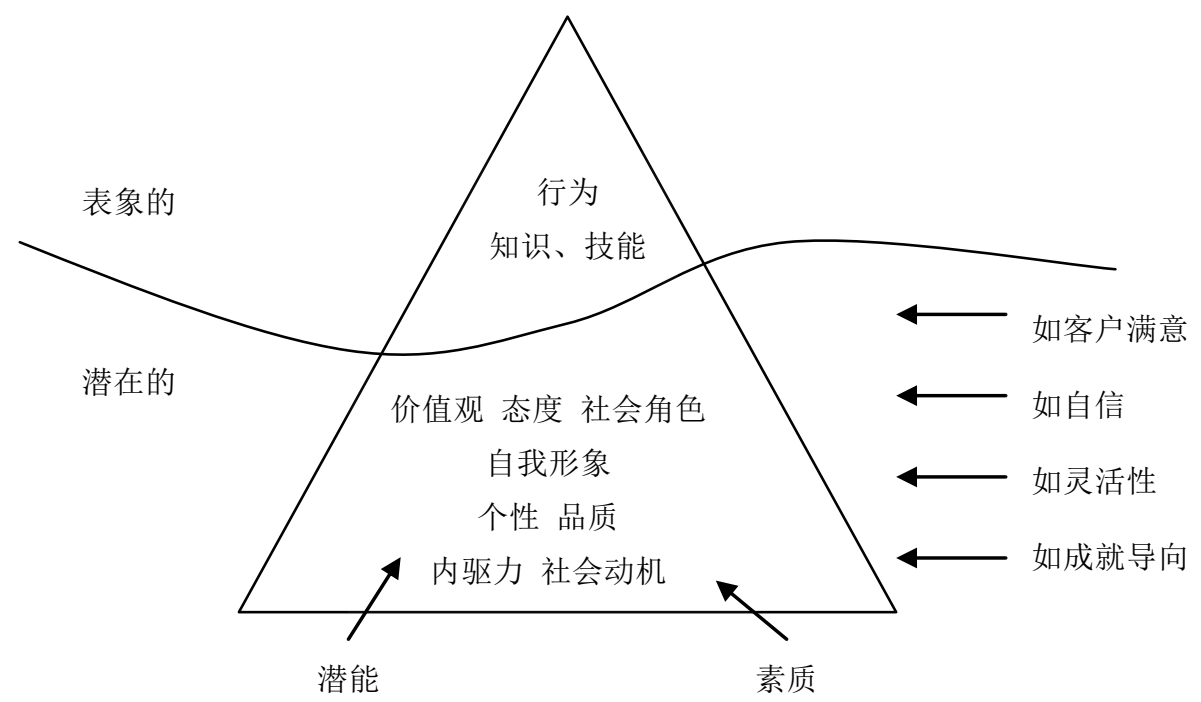

图 1 麦克利兰素质冰山模型

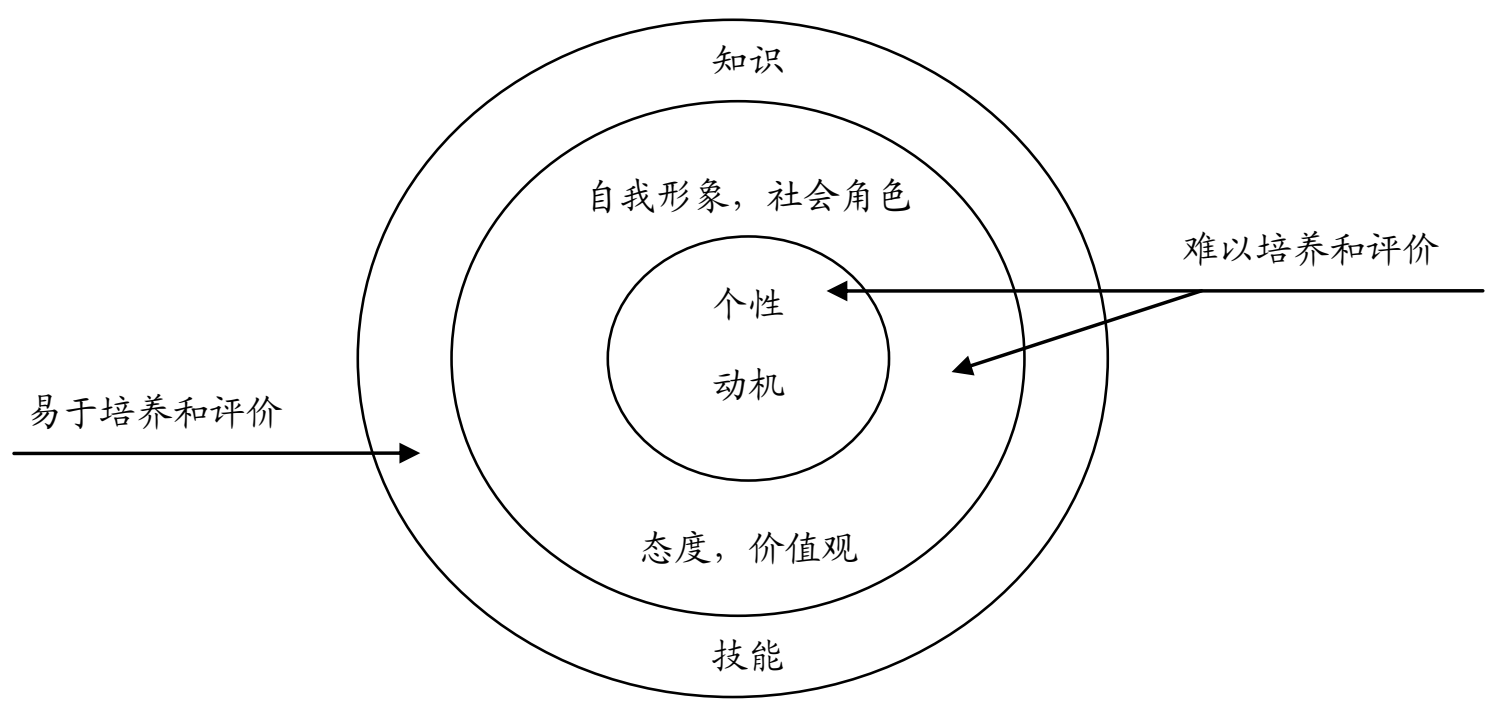

图 2 博亚特兹素质洋葱模型

美国学者博亚特兹对麦克利兰的能力素质理论进行了 深入和广泛的研究。提出了洋苟模型, 展示了能力素质构 成的核心要素, 并说明了各构成要素可被观察和衡量的特 点。该模型如图 2 所示。基于以上对能力素质模型的分析, 可以得出能力素质模型具有两个核心要点: 一是能力素质 是可以观察和衡量的。如果能力素质只是建立在经验之上 不具有可观察性, 那么这样的能力素质是不具有说服性的, 甚至是不科学的。二是能力素质的确定及其指标的测量都 必须建立在客观依据之上。尤其是指标的测量必须要有科 学性和说服力, 否则是不能成立的。
(2) 能力素质模型的特点

能力素质模型的特征主要有以下三个方面:

首先, 具有行业特色。它反映的是某类行业内对人员 的整体素质要求, 包括知识和技能的范围, 对所服务客户 的认识程度等。

其次, 具有组织特色。它反映的是单个组织对特定人 员的要求, 并且细化到行为方式的程度, 即使是处于同一 行业的两个组织, 由于组织文化、目标、策略的差异, 纵 然组织在人员要求的能力条目上完全相同, 也很少有两个 组织的能力素质的行为方式要求是完全一致的。 
最后, 具有阶段性。能力素质模型的行为模式由于与 组织运作相关联, 因而具有阶段性。在组织的特定时期内, 某项胜任能力, 甚至是某一组能力是至关重要的, 而在另 一个阶段, 由于组织的目标或策略发生变化, 能力素质模 型就会定期随之更新和改变。

\section{3. 基于能力素质模型的研究生拔尖创新人才能力素 质分析}

\section{1 相关概念的界定}

\subsection{1.拔尖创新人才}

本文认为拔尖创新人才作为我们的教育理想, 意指在 我们所生活的这个富于个性和突破的时代中, 在各行各业 那些试图通过变革来引领发展, 从而为整个社会经济的顺 利转型做出突出贡献的杰出人物, 精深的专业造诣、强烈的 社会责任感以及勇于批判和变革的勇气应作为其基本的素 质特征。

\subsection{2. 能力的内涵}

能力是具有主动性的人或者物所具有的综合素质。能 力的拥有者一般是具有生命的人或者生物。但是, 从组织 能力的角度来说, 能力一般指的是组织长期积累的知识、 文化等的总和。本文所指的能力是主要从个人胜任力的角 度去理解。

\section{2 相关文献分析}

有关研究生拔尖创新人才的能力构成学术界已有相关
研究。卜祥云等 (2008) 认为高校拔尖创新人才能力特征 主要表现在: 知识渊博, 个性突出, 善于创新, 开拓进取, 追逐卓越, 实现自我, 苛求认同, 期望尊重几个方面。马 一博（2010）运用马兹罗的创新能力表现形式分类理论对 经管类研究生创新能力表现形式进行分类, 通过建立创新 能力指标体系对经管类研究生创新能力构成进行分析。关 华等 (2011) 结合中科院数学院的实际, 提出拔尖创新人 才的能力应包括: 掌握本学科坚实宽广的基础理论和系统 深入的专门知识的能力, 独立从事科学研究工作的能力, 在科学研究和专门技术等方面做出创造性成果的能力。林 崇德 (2011) 从心理学的角度指出自然科学创新人才的重 要心理特征主要包括内部驱动的动机形式、面向问题解决 的知识构架、自主牵引性格、开放深刻的思维与研究风格、 强基础智力等五个因素; 哲学社会科学和艺术领域的创新 人才, 与自然科学创新人才相比, 心里特征突出表现在人 格方面。

\section{3 研究生能力素质 “冰山” 模型}

根据能力素质模型理论和相关研究的最新成果, 以 “冰 山” 模型理论为基础, 在对研究生能力素质文献分析、研 究生培养目标和培养模式分析的基础上, 经过去除重复项、 合并相似项、去除描述过于泛化的项目等一系列归纳总结, 结合咨询访谈, 初步提出研究生拔尖创新人才需具备的能 力特征。研究生拔尖创新人才能力特征至少应具备品质与 个性、知识、技能等个方面:

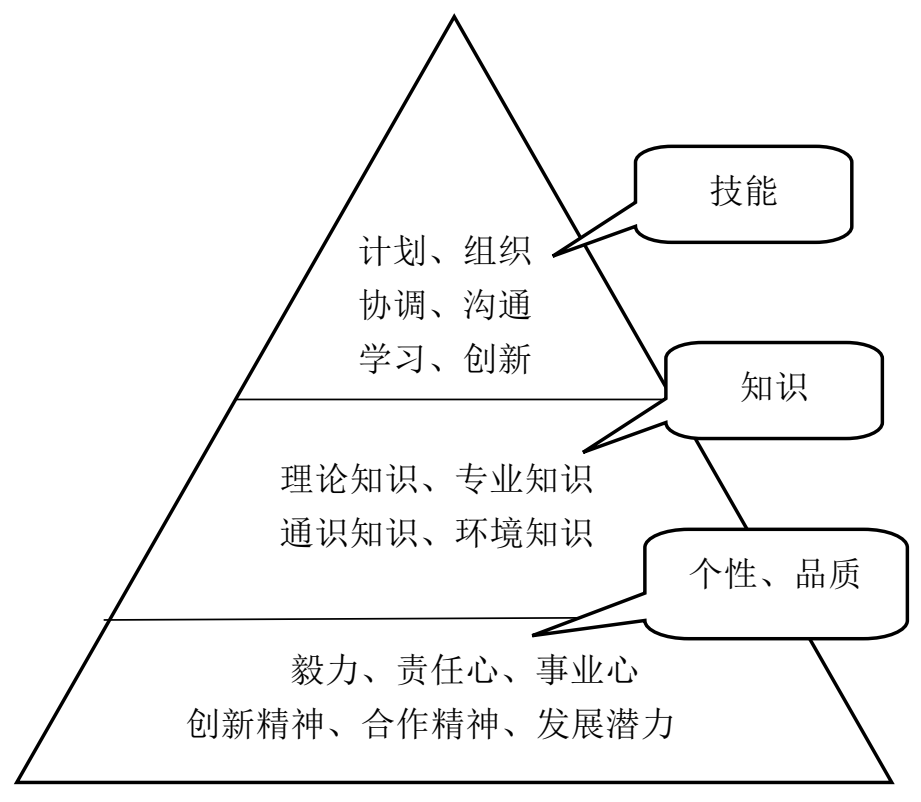

图 3 研究生能力素质 “冰山” 模型 


\section{4. 结束语}

本文以能力素质模型为理论基础, 对研究生拔尖创新 人才所应具备的能力素质进行了分析, 在对研究生能力素 质文献分析基础之上, 结合专家访谈, 归纳总结出研究生 拔尖创新人才能力特征至少应具备知识、技能、品质、个 性等四个方面, 并依据能力素质 “冰山” 模型, 建立了研 究生拔尖创新人才能力素质冰山模型, 为后续的研究生拔 尖创新人才能力测评奠定基础。

\section{参考文献(References)}

[1] William J. Rothwell, John E. Lindholm, Competency identification, modeling and assessment in the USA, International Journal of Training and Development,Vol.3, pp.90-105, 1999.

[2] Deng XiuQuan, Kang Yun Peng, Xi Jun Feng, Bai Bing , Mao Yang Xu. Research on resource competence model of research team in university and its application. Studies in Science of Science. vol. 30, pp. 102-110, 2012.

[3] Ma Yibo. Study on innovative ability evaluation system about economics and management graduates. Harbin Engineering University Dissertation.2010

[4] Bu Xiangyun, Tang Guiwu, Cai Xiang. On college Elite competency and performance. Science and Technology Management Research. vol. 28, pp. 323-324, 2008.

[5] Bradley, Elizabeth H.; Cherlin, Emily, Adopting a Competency-Based Model; Mapping Curricula and Assessing Student Progress, Journal of Health Administration Education, Vol.25, pp. 37-51, 2008.

[6] Guan Hua, Shao Xin, Wang Shouyang. Innovation pattern and multiple training for top-notch innovative talents. Bulletin of the Chinese Academy of Sciences. vol. 26, pp. 237-2404, 2011.

[7] Lin Chongde. Ways to cultivate innovation ability from the perspective of the characteristics of creative talent. Scientific Chinese. vol. 9, pp. 18-21, 2011. 